\title{
ArcheoSciences
}

Revue d'archéométrie

$34 \mid 2010$

Varia

\section{Une mine de plomb argentifère dans un environnement montagnard}

La mine médiévale du Fournel à L'Argentière-la-Bessée (Hautes-Alpes)

A silver-bearing lead mine in a mountainous environment: The medieval Fournel mine at L'Argentière-la-Bessée (Hautes-Alpes)

Bruno Ancel, Vanessa Py, Eric Kammenthaler, Vincent Leleu et Christophe Marconnet

\section{(2) OpenEdition}

\section{Journals}

Édition électronique

URL : https://journals.openedition.org/archeosciences/2771

DOI : 10.4000/archeosciences. 2771

ISSN : 2104-3728

Éditeur

Presses universitaires de Rennes

Édition imprimée

Date de publication : 10 avril 2010

Pagination : 203-220

ISBN : 978-2-7535-1407-2

ISSN : $1960-1360$

Référence électronique

Bruno Ancel, Vanessa Py, Eric Kammenthaler, Vincent Leleu et Christophe Marconnet, « Une mine de plomb argentifère dans un environnement montagnard », ArcheoSciences [En ligne], 34 | 2010, mis en ligne le 11 avril 2013, consulté le 01 février 2022. URL : http://journals.openedition.org/ archeosciences/2771 ; DOI : https://doi.org/10.4000/archeosciences.2771 


\title{
Une mine de plomb argentifère dans un environnement montagnard : la mine médiévale du Fournel à L'Argentière-la-Bessée (Hautes-Alpes)
}

\author{
A silver-bearing lead mine in a mountainous environment: \\ The medieval Fournel mine at L'Argentière-la-Bessée (Hautes-Alpes)
}

\author{
Bruno AnCel*, Vanessa Py**, Christophe Marconnet ${ }^{* * *}$, \\ Eric KammenthaleR ${ }^{* * * *}$ et Vincent Leleu*
}

\begin{abstract}
Résumé : La mine d'argent du Fournel a été en activité entre le $\mathrm{x}^{\mathrm{e}}$ et le XIV siècle. Les textes conservés concernent la phase de maturité du $\mathrm{XII}^{\mathrm{e}}$ siècle et la phase de ralentissement du milieu du XIII ${ }^{\mathrm{e}}$ siècle. Plusieurs panneaux filoniens ont été exploités à partir d'affleurements, depuis des escarpements rocheux abrupts, jusqu'au fond d'une gorge. La grande dureté de la roche a obligé les mineurs à recourir à l'abattage par le feu. Le relief a grandement facilité la prospection des filons, le démarrage de chantiers à ciel ouvert et l'organisation des exploitations souterraines. Les chantiers s'étendent jusqu'à $150 \mathrm{~m}$ du jour et sont assistés par des niveaux de circulation par traînage, des conduits d'aérage perchés en hauteur et des galeries de drainage ouvertes à la base. Ces ouvrages d'assistance reflètent une organisation opportuniste de la mine, facilitée par la topographie et la géologie du gisement. L’analyse sédimentologique et anthracologique des remblais, associée à des expérimentations sérielles, permet de caractériser la chaîne opératoire technique de l'abattage par le feu et son impact sur les modalités de gestion de la forêt arborescente d'altitude, comprimée entre les activités agropastorales et minières.
\end{abstract}

\begin{abstract}
The Fournel silver mine was in operation between the tenth and fourteenth century. The extant texts concern the twelfth century mature phase and the mid-thirteenth century slowdown. Several vein panels were exploited from outcroppings, in steep rocky cliffs, to the bottom of a gorge. Because of the extreme hardness of the rock, the miners used firesetting. The topography of the site greatly facilitated vein exploration, initiating opencast sites and mine organisation. The stopes stretch up to $150 \mathrm{~m}$ from daylight, and were assisted by access levels by sledge, high perched ventilation ducts and drainage adits open to base level. These works reflect an opportunist support organisation in the mine, facilitated by relief and deposit geology. Sedimentological analysis of backfill and the use of anthracology associated with serial experiments, enable characterisation of the operative chain techniques of firesetting and its impact on high altitude forest management up to tree level, between the higher pastures and the mines.
\end{abstract}

Mots clé : abattage par le feu, Alpes, gestion forestière, médiéval, mine, stratégie d'exploitation.

Key words: Alps, firesetting, forestry management, medieval, mining, working strategy.

\footnotetext{
* Service Culturel Municipal - Hôtel de Ville, 05120 L'Argentière-la-Bessée.

** Université de Provence, Aix-Marseille I, LAMM UMR 6572 CNRS.

Maison Méditerranéenne des Sciences de l'Homme - 5, rue du Château de l'Horloge, BP 647, 13094 Aix-en-Provence cedex 2.

*** Arkémine Mine de Rosiers 63230 Saint-Pierre-le-Chastel.

**** Iker Archéologie - Maison Errekaldia 64780 Saint-Martin-d'Arrossa.
} 
L'activité minière du site du Fournel a cessé en 1908. L'établissement moderne a été ravagé par des crues, puis livré au pillage dans les années 1930 . Tombée dans l'oubli, connue seulement par quelques géologues et spéléologues, la mine a refait surface en 1991 en faisant l'objet d'un programme de recherche scientifique et de mise en valeur touristique, accompagné de la création d'un service du patrimoine au sein de la commune de L'Argentière-laBessée (Ancel, Cowburn, 1998; Cowburn, 2008).

Les vestiges de l'exploitation médiévale et des reprises du XIX ${ }^{e}$ siècle (1785 à 1908) ont été prospectés, relevés, et en partie fouillés, sous la direction scientifique de Bruno Ancel, de 1992 à 2007 (Ancel et al., 1996; Ancel, 2006a). Cette recherche s'est effectuée sur l'ensemble du secteur minier, mais surtout au fond des gorges du Fournel où le développement touristique a généré des moyens humains et financiers qui ont dynamisé les travaux archéologiques.

Un premier bilan sur l'exploitation médiévale a été dressé lors du congrès de Civezzano-Fornace en 1995 : les différents vestiges ont alors été décrits en détail (Ancel, 1998a). L'approche anthracologique démarrée en 2001 par Vanessa Py a été l'occasion de relancer l'étude des ouvrages anciens, des remblais miniers et de la technique d'abattage par le feu (Py, 2009). Cette recherche a été soutenue à partir de 2006 par l'ACI « Histoire des savoirs » coordonnée par Aline Durand : Savoir brûler, Savoir gérer le combustible chez les potiers et les mineurs méridionaux (XI'-XVI siècles) (Durand, 2007).

La présente synthèse a pour objectif de dresser un bilan des connaissances actuelles sur l'exploitation médiévale du Fournel en relevant les incertitudes et les points d'ombre liés à la représentativité différentielle des vestiges plus ou moins bien conservés et dont l'investigation archéologique a été plus ou moins approfondie.

\section{LA MINE DANS SON ENVIRONNEMENT NATUREL ET HISTORIQUE}

\section{Le cadre géologique}

La géologie de la mine est bien connue grâce à la documentation XIx ${ }^{e}$ siècle du Service des Mines et aux études récentes menées par le BRGM sous la coordination de Jean Feraud (BRGM, 1997; 2004).

\section{Le minerai}

Le minerai exploité était de la galène argentifère, un sulfure de plomb (PbS) renfermant 2 à $3 \%$ d'argent. Cette galène est localement accompagnée de blende $(\mathrm{ZnS})$ et de minéraux métalliferes accessoires : « cuivre gris » (tétraédrite, tennantite), pyrite et/ou chalcopyrite. Ces différents minerais de cuivre, zinc et fer ont des teneurs totalement inexploitables. La galène est mélangée à une gangue de quartz et de barytine, et parfois, elle imprègne la roche encaissante.

La minéralisation avait une puissance de quelques centimètres à plusieurs mètres $(8 \mathrm{~m}$ dans le quartier Centre de la reprise moderne, d'après les archives). Dans les parties exploitées au Moyen Âge la puissance était de $10 \mathrm{~cm}$ à $2 \mathrm{~m}$. Schématiquement, le minerai se concentre souvent dans une bande massive où la galène représentait 15 à $25 \%$ de la masse, la barytine, 15 à $50 \%$, le quartz, 20 à $70 \%$ et la blende, 0 à $7 \%$. Cette bande massive avait souvent une épaisseur de 20 à $30 \mathrm{~cm}$, mais localement elle pouvait atteindre $1,50 \mathrm{~m}$. Dans la partie pauvre du filon, la galène prenait la forme de minces veines ou de manière diffuse, et elle ne représentait que quelques pourcents de la masse.

\section{L'encaissant}

La minéralisation est encaissée dans une formation de quartzites. Il s'agit d'anciens sables marins d'âge werfénien (base du Trias, ère secondaire, environ 250 millions d'années), d'abord transformés en grès blanc, puis recristallisés en quartzites sous l'effet d'un faible métamorphisme lors de la surrection alpine. Cette formation puissante de $300 \mathrm{~m}$ repose sur des conglomérats à faciès Verrucano (PermoTrias). Elle est surmontée par environ $20 \mathrm{~m}$ de schistes gris ou noirs, puis par plusieurs centaines de mètres de calcaire massif (Trias moyen). Comme la gangue du filon est souvent très quartzeuse, il n'est pas évident de la distinguer de la roche encaissante au premier coup d'oeil. Par contre, le minerai étant gris noir, il est facilement repérable à l'affleurement, mais pas sur les parois salies des travaux souterrains.

\section{Le gisement}

La minéralisation remplit un filon dont l'étendue d'origine devait avoisiner $0,5 \times 1 \mathrm{~km}$, avec des zones d'étranglement qui définissent plusieurs secteurs bien minéralisés. Ce filon correspond à un plan de chevauchement associé au charriage des nappes briançonnaises à la fin de l'Éocène (35 millions d'années). Le mouvement de chevauchement n'est que de $50 \mathrm{~m}$. Il affecte l'ensemble des formations sédimentaires qui ont été redressées à la verticale par un plissement précédent (fig. 1). Ainsi, du côté Ouest, les quartzites et la minéralisation chevauchent les schistes noirs, alors que du côté Est, c'est le Verrucano qui chevauche le filon et les quartzites. À la fin de l'Oligocène (25 millions d'années) une nouvelle phase de compression voile l'ensemble de la structure. Au Pliocène (4 millions d'années) le filon est découpé par un jeu de faille en panneaux métriques à décimétriques, décalés les 


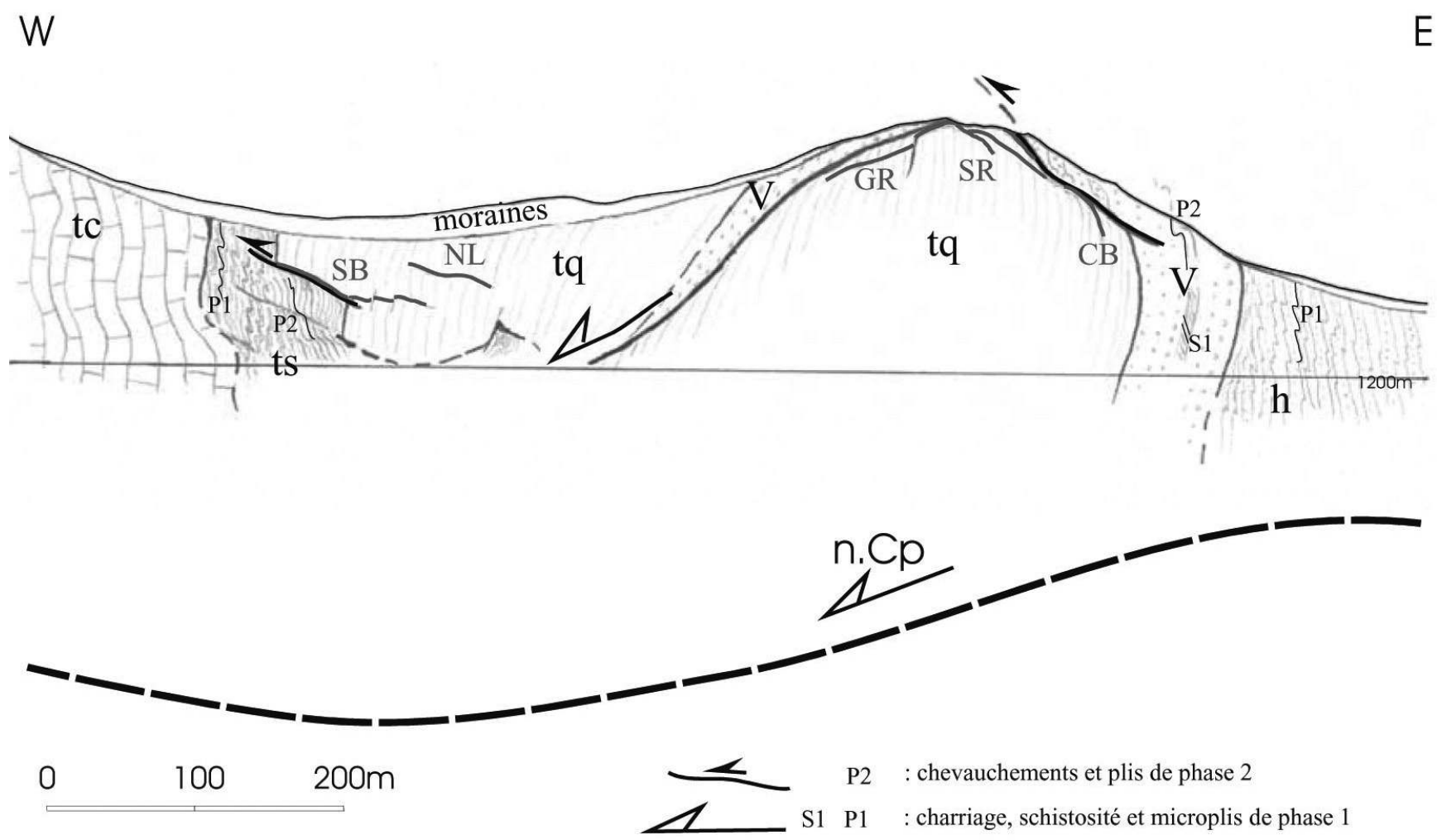

Figure 1 : Coupe géologique synthétique du gîte du Fournel.

Figure 1: Synthetical geological section of the Fournel vein deposits.

uns par rapport aux autres par des rejets de quelques mètres à plusieurs dizaines de mètres (surtout au Gorgeat).

\section{Les conséquences minières}

Au XIx ${ }^{e}$ siècle, la quasi-totalité du gisement a été reconnue grâce au percement de plusieurs kilomètres de galeries de recherche avec une progression moyenne de $50 \mathrm{~m}$ par an. Grâce aux appareils de préparation mécanique du minerai permettant l'enrichissement de la partie pauvre de la minéralisation, sa totalité a été extraite (Ancel, 2006).

Au Moyen Âge, l'exploitation s'est limitée aux panneaux qui affleuraient en surface. La dureté de la roche a interdit les longs travaux de recherche (progression de $5 \mathrm{~m}$ par an). Quelques rejets de faille de faible ampleur ont pu être franchis. Seule la bande massive de minerai a été exploitée. Le minerai abattu et trié ne pouvait guère dépasser une teneur de $35 \%$ et n'offrait aucun intérêt en tant que colorant ou poudre de vernissage (alquifoux).

\section{Le cadre géographique}

Après la surrection des Alpes, le relief s'est progressivement mis en place durant le Pléistocène, avec notamment quatre phases glaciaires qui ont profondément marqué le paysage. Le gisement du Fournel a été mis au jour par l'érosion, à la confluence du vallon du Fournel avec la vallée de la Durance. Après le retrait des glaces, il y a 12000 ans, le torrent du Fournel a entaillé les $250 \mathrm{~m}$ de dénivelé qui le séparaient du lit de la Durance, en creusant une gorge escarpée dans les quartzites et un canyon étroit dans les calcaires. De ce fait, les affleurements de minerais sont bien visibles depuis les hauteurs qui dominent L'Argentière (crête de Saint-Roch) jusqu'au fond des gorges (Gorgeat, Lauzebrune). Par contre, à mi-hauteur, sur une banquette correspondant au fond de l'ancienne vallée glaciaire, une épaisse formation de moraines masque la roche encaissante et la minéralisation. Elle n'y sera découverte que vers 1870 à la suite du percement d'un long cheminement souterrain sous le versant.

L'ensemble du secteur minier est facilement accessible pour des populations montagnardes. Les escarpements rocheux sont peu végétalisés et l'érosion est toujours active. Le minerai sombre était bien visible sur la roche claire et devait être parfaitement connu des bergers. Pour les exploitants du XIX ${ }^{e}$ siècle, l'escarpement du site fut par contre un problème pour la construction d'un établissement et pour le transport des matériaux (Ancel, 2000; 2008b). 


\section{Le cadre historique}

La mine située dans le ressort banal du castrum de Argenteria est mentionnée explicitement dans la première enquête delphinale de 1250. Le dauphin, qui est enfin parvenu à étendre ses prétentions sur la Haute Durance, y légiferre l'activité minière en vertu de son plenum dominium dans une courte notice réglementaire. Il contrôle l'ouverture et la déshérence des fosses minières dans la propriété foncière d'autres. Chaque croterius ou cros devait mesurer cinq toises sur le côté (in latere), en d'autres termes, pouvait se développer sur une emprise (ou un diamètre) de 19,5 m, soit une aire totale de $300 \mathrm{~m}^{2}$, la profondeur n'étant pas stipulée. En plus de la redevance du vingtième sur la production d'argent, le dauphin jouissait d'un droit de préemption sur le négoce du métal par les producteurs et percevait une taxe de six deniers levée pro dominio suo sur la vente de chaque calice fabriqué par les orfevres du mandement. Cette taxe implique l'existence d'ateliers de transformation dans le mandement de L'Argentière au milieu du XIII ${ }^{\mathrm{e}}$ siècle.

Ce texte est le seul et le dernier à attester directement une activité minière à L'Argentière. Les archives seigneuriales conservées à partie de 1260 ne mentionnent plus jamais la mine dont l'activité s'est ralentie, voire éteinte. Au regard de l'ampleur des vestiges archéologiques, ces données sont extrêmement laconiques. De plus, le phasage chronologique de l'exploitation, obtenu grâce à une importante batterie de datations radiocarbones couplée avec une caractérisation anthracologique des dépôts soumis à l'analyse, atteste son démarrage au $\mathrm{X}^{\mathrm{e}}$ siècle et son intensification au $\mathrm{XI}^{\mathrm{e}}$ siècle. La reprise complète du corpus documentaire a permis de préciser l'histoire de l'activité minière avant le XIII ${ }^{\mathrm{e}}$ siècle (Py, 2009). Dans une bulle pontificale datée de 1150, l'Église d'Embrun se fait confirmer en même temps que d'autres possessions, la troisième partie des mines d'argent situées dans les lieux de Rame, Champcella, Freissinières et Erego. Elle provient d'une donation réalisée par Giraldus Malvicinus au milieu du $\mathrm{XI}^{\mathrm{e}}$ siècle. Les mines réapparaissent dans une bulle pontificale datée de 1169 , destinée à régler les conflits qui ont éclaté au sein de l'Église d'Embrun au sujet de la répartition des revenus miniers des argenteria de Erego et Faravello entre l'archevêque, son chapitre et le prévôt. Les mines désignées dans la bulle sont clairement identifiées comme étant celles de Faravel, district satellite du Fournel situé dans la vallée de Freissinières, et celles de L'Argentière située dans un lieu alors dénommé Erego. Parallèlement, le comte d'Albon reçoit en fief en 1155, de l'empereur Frédéric Ier, détenteur du droit régalien, la mine d'argent située dans le ressort banal de Rame et dans la juridiction des comtes de Provence et de Forcalquier, autrement dit la mine d'Erego.
Ces textes indiquent que la troisième partie de la propriété des mines et des terrains appartenait à l'Église d'Embrun depuis le $\mathrm{XI}^{\mathrm{e}}$ siècle. Elle s'était aussi fait reconnaître la dîme des métaux dans l'étendue de son diocèse en 1147 par l'empereur Conrad III. Les deux autres parties étaient manifestement restées dans les mains des héritiers de Giraldus très liés à l'Église d'Embrun et faisant partie de la clientèle des comtes de Provence et de Forcalquier. Le comte d'Albon, vassal de l'empereur, avait quant à lui obtenu en fief le droit régalien de faire exploiter les mines et de prélever une part des revenus. Il acquiert la haute juridiction comtale du castrum de L'Argentière seulement en 1232, mais en tant que vassal de l'archevêque d'Embrun.

On se trouve devant une stratification typiquement médiévale de différents pouvoirs exercés principalement par des seigneurs locaux. La documentation conservée ne permet malheureusement pas de préciser les rapports entre l'Église d'Embrun et les comtes de Provence et de Forcalquier entre le $\mathrm{XI}^{\mathrm{e}}$ et le début du XIII ${ }^{\mathrm{e}}$ siècle, ni même entre l'Église d'Embrun et le dauphin au XIII ${ }^{\mathrm{e}}$ siècle.

\section{L'impact des reprises modernes}

Vers 1670 et 1740 , deux tentatives de reprise sont signalées. De 1788 à 1793, puis de 1835 à 1838, deux phases d'activité, assez bien documentées, concernent la reprise des anciens travaux (Ancel, 2006b). Avant la découverte de nouveaux panneaux minéralisés, les mineurs s'échinent à récupérer le maximum de minerais dans les ouvrages médiévaux. Ainsi, les anciens travaux sont explorés, les fronts de taille sont rafraîchis, des passages sont aménagés au sein des chantiers comblés et des piliers de minerais sont abattus. Ces reprises, souvent accompagnées de dépilages déraisonnés, ont conduit au chamboulement des parties anciennes les plus accessibles et à l'effondrement de plusieurs chantiers.

Les rapports techniques de l'époque décrivent succinctement les ouvrages anciens et leurs principales caractéristiques - travaux ouverts par le feu - et de ce fait L'Argentière est souvent citée dans la littérature comme un important témoignage de l'activité minière des Anciens. Bien que la plupart des textes médiévaux aient été déjà publiés, les préjugés de l'époque lui attribueront une origine romaine qui persistera jusqu’à nos jours (Ancel, 2006b).

\section{LES VESTIGES EN SURFACE}

\section{Les sites d'extraction}

L'escarpement des lieux permet une bonne visibilité des sites d'extraction et de nombreux orifices sont restés ouverts. 
Cependant, en pied de falaises, les accumulations d'éboulis ont pu faire disparaitre certaines entrées. Au fond des gorges, les surcreusements et les engravements du lit du torrent font que certains orifices se retrouvent aujourd'hui perchés en falaise et que d'autres sont totalement obturés par des alluvions. Tous les lieux de travaux anciens mentionnés au XIX $x^{e}$ siècle ont été retrouvés et des sites inédits ont également été découverts. On distingue ainsi sept quartiers de travaux anciens : au fond des gorges, le Gorgeat et Lauzebrune; en rive droite, l'Albret; et sur les escarpements dominant L'Argentière, Combe Blanche, Saint-Roch, la Rouille et la Pinée (fig. 2).

Il s'avère que tous les affleurements de la minéralisation, même stériles, ont été reconnus par les Anciens. Le moindre indice présente des traces de grattages. Les zones productives ont été attaquées à ciel ouvert. Lorsque la minéralisation est sécante avec la surface, les chantiers ont pris la forme de tranchées verticales ou obliques, rapidement obstruées par des éboulements quand la roche encaissante est fracturée. Lorsque la minéralisation est parallèle à la surface - on dit qu'elle affleure en surface structurale - il en résulte une exploitation à ciel ouvert semblable à une carrière qui peut se prolonger sur les bords par un chantier souterrain. Ce cas de figure assez exceptionnel se présente au Fournel sur trois quartiers où environ $2400 \mathrm{~m}^{2}$ de minéralisation ont pu être très facilement extraits. Cet atout gîtologique a très certainement favorisé le démarrage de l'activité.

Sur les affleurements stériles ou à proximité immédiate des exploitations, on recense vingt-cinq entrées de galeries et quinze ouvertures de puits ou descenderie dont certaines sont actuellement masquées, mais sont localisées à partir des données souterraines. Il demeure difficile d'évaluer l'existence d'autres entrées situées à l'écart des filons dans des zones gagnées par les éboulis.

Les orifices principaux de l'exploitation souterraine sont le point de départ de vastes épandages de déblais, appelés haldes. Ils sont extrêmement développés sur le versant de la Durance (Rouille, Pinée) où leur couleur rougeâtre tranche nettement sur la grisaille des éboulis naturels. Mais, leur étalement sur la pente abrupte ne permet plus aujourd'hui de distinguer clairement les replats qui auraient pu exister à l'origine. Dans les zones escarpées, ces épandages sont érodés et lessivés. Ainsi, le ravin de Combe Blanche doit certainement son nom aux déblais miniers qui ont dû le tapisser autrefois. Dans les gorges, les crues ont fait disparaitre toute trace d'activité minière en surface.

\section{Les aires de traitement}

Le contexte géographique et la topographie n'ont pas favorisé la conservation des aires d'occupation aux abords des sites d'extraction. Le temps a fait son oeuvre, soit en érodant ces aires, soit en les recouvrant d'éboulis ou d'alluvions torrentielles.

Potentiellement, le minerai devait subir un minimum de traitement manuel à sa sortie au jour, c'est-à-dire un scheidage comprenant un cassage et un tri à la main, pour ne garder que du minerai "riche" à 20-35\% de galène. La fouille d'une entrée de galerie à Combe Blanche a livré une petite plateforme encastrée dans la pente rocheuse renfermant deux enclumes de cassage et quelques dépôts de gangues triées et calibrées. D'autres enclumes en pierre dure ont été retrouvées en réemploi dans les bâtiments XIx ${ }^{e}$ siècle du Gorgeat. Dans les escarpements rocheux, il existe plusieurs sites potentiels d'aire de traitement, mais leur fouille nécessiterait d'évacuer d'importants volumes d'éboulis et de blocs sur une pente raide qui domine une route, un canal d'irrigation et des habitations.

Il n'est pas impossible que les mineurs aient poursuivi l'enrichissement du minerai par un broyage et lavage à l'eau. Mais, il serait vraiment fortuit de retrouver des installations de lavage au bord du torrent.

\section{La question de la métallurgie}

Le minerai devait ensuite subir des opérations de grillage, puis de réduction, et enfin d'affinage pour séparer l'argent du plomb. Ces opérations métallurgiques produisaient des déchets, ou scories, assez abondants. La mine de L'Argentière a potentiellement pu produire plusieurs milliers de tonnes de scories, mais pas une seule n'a été retrouvée sur les versants de la mine, dans la plaine alluviale ou autour du site castral d'Urgon.

Il est possible toutefois d'émettre deux hypothèses qui ne sont pas incompatibles. Une métallurgie itinérante a pu se pratiquer dans les massifs boisés d'altitude du mandement de L'Argentière. Des charbonnières datées des $\mathrm{XI}^{\mathrm{e}}$-XII ${ }^{\mathrm{e}}$ siècles par l'analyse radiocarbone ont été localisées dans le bois du Simon de l'Aigle à plus de 1800 m d'altitude (Py, 2004). L'activité métallurgique a pu aussi être centralisée, d'une part près de l'atelier monétaire des archevêques d'Embrun dans le territoire de Ceillac, et, d'autre part, près de l'atelier monétaire du dauphin à Césane en Piémont (Cesana Torinese). Pour l'instant aucune découverte archéologique fiable ne permet d'être catégorique à ce sujet.

\section{LES VESTIGES SOUTERRAINS}

Près de $2 \mathrm{~km}$ de travaux souterrains anciens ont pu être explorés (fig. 2). Comparativement aux autres exemples de 
travaux anciens à ciel ouvert

travaux anciens explorés

travaux anciens supposés

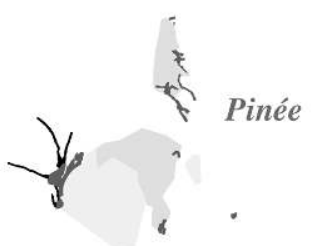

travaux modernes explorés

travaux modernes connus par les archives

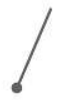

$4 \quad$ Rouille
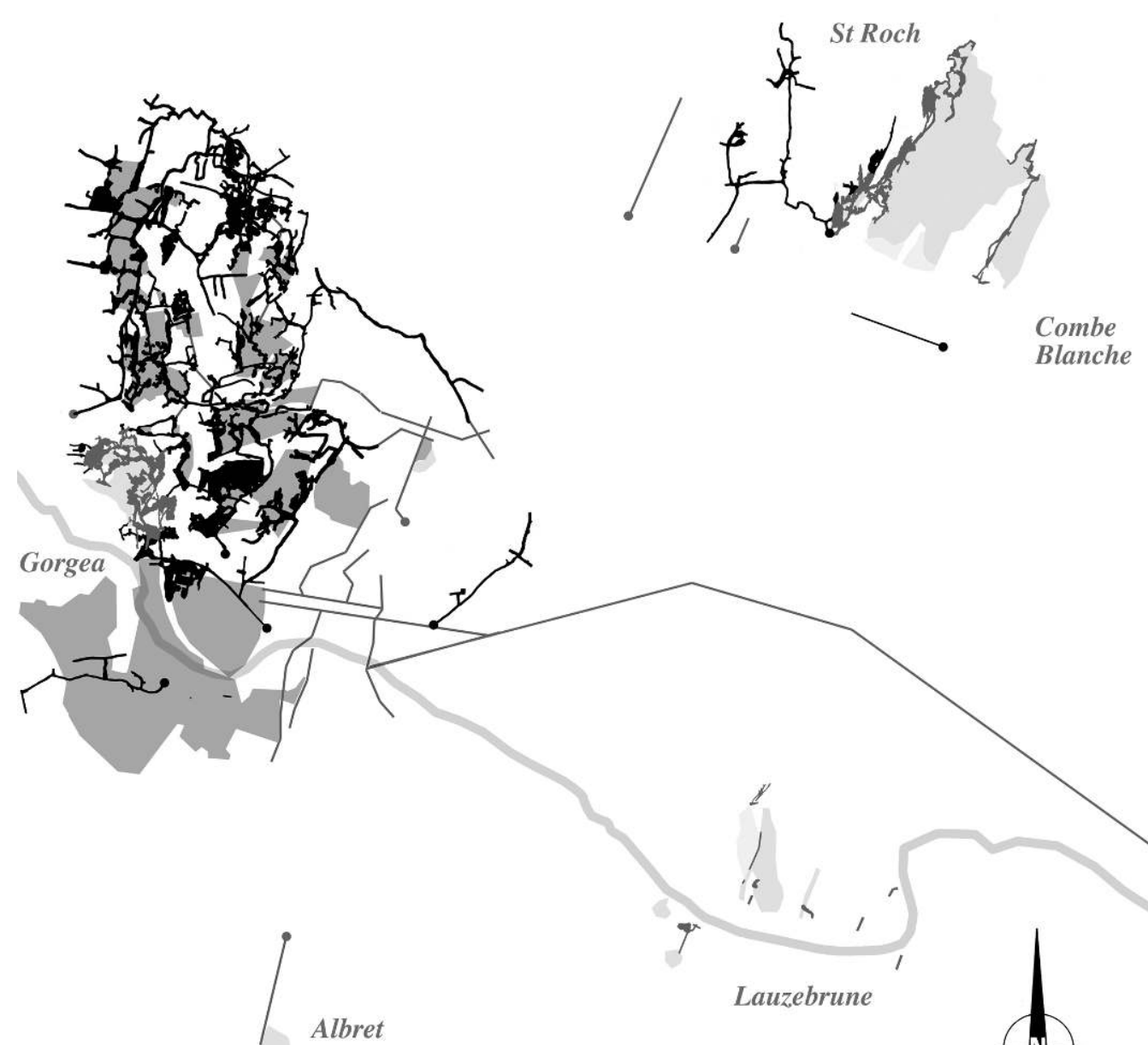

B. Ancel 2000

$100 \mathrm{~m}$

Figure 2 : (Voir planche couleur) Carte souterraine des travaux miniers du Fournel.

Figure 2: (See colour plate) Map of the Fournel underground workings. 
mines de l'Occident médiéval, la mine du Fournel est relativement bien conservée et ses vestiges sont assez représentatifs.

\section{Les ouvrages}

Les vides creusés par les mineurs de L'Argentière concernent à $99 \%$, l'abattage de la minéralisation : ce sont les chantiers d'exploitation. Leurs allures sont dictées par la géométrie du filon (vertical, incliné, plat), la puissance de la minéralisation (exigu, spacieux) et l'étendue des panneaux filoniens (quelques mètres à plus de $100 \mathrm{~m}$ ). Ils sont généralement fortement remblayés, donc difficiles à explorer, à observer et à évaluer. Lorsque la fouille permet de les vider de leurs remblais, on observe généralement des voûtes en coupoles résultant de l'abattage par le feu, des sols en gradins irréguliers induits par la géologie (failles, stratification des quartzites) et des fronts de taille pincés ou arrondis selon la méthode de taille.

Les ouvrages d'assistance (Ancel, 1998b) sont limités en extension et très proches des chantiers. Dans Saint-Roch, plusieurs branches minéralisées sont exploitées par des chantiers inclinés parallèles. Ils sont reliés entre eux par des puits peu profonds, creusés sur des failles. Les affleurements sont souvent suivis par des puits redressés ou des descenderies en gradins. Ces ouvrages verticaux ne présentent pas de trace d'aménagements (encoche de boisage, aire de treuillage).

La plupart des galeries sont creusées sur des zones de failles et sont étroites. Leur section influencée par la géologie est souvent quadrangulaire et non pas ovoïde comme le sont généralement les galeries médiévales du massif vosgien (Grandemange, 1990; Fluck, 2000). Leur véritable hauteur est masquée par des remblaiements tardifs. Contrairement aux premières impressions, leur fouille montre que celles qui ont pu servir à la circulation sont assez régulières et suffisamment hautes.

\section{Les techniques}

\section{L'abattage}

La technique d'abattage employée dépend de la dureté de la roche. Dans une zone de faille où la roche est broyée sur plusieurs décimètres d'épaisseur, des traces de pointerolles sont parfaitement conservées. Lorsque la roche est très fracturée, comme au contact des schistes noirs, l'abattage a sans doute été manuel, mais les traces d'outils n'y sont pas conservées.

En roche dure, l'abattage par le feu prédomine. Les parois des ouvrages montrent souvent des surfaces lisses et arrondies, mais il ne faut pas oublier qu'une phase de purge manuelle succède toujours à la phase d'étonnement due à la montée en température de la roche. Aussi, une fracturation légère de la roche peut rendre les parois plus irrégulières (Ancel \& Py, 2008). Lorsqu'il existe une zone de faiblesse dans la roche - une zone broyée ou une abondance de barytine dans le filon - elle est attaquée à la pointerolle sous la forme d'une entaille qui est ensuite élargie au moyen du feu. Cette technique mixte explique la forme pincée des fronts de taille de certains chantiers. Une sorte de fourche en fer découverte au Gorgeat pourrait correspondre à un outil de purge des fronts de taille.

Si les ouvrages d'assistance sont généralement creusés sur des zones de faiblesse, il existe néanmoins des exemples ouverts par le feu en roche très dure. À Lauzebrune, un travers-bancs d'exhaure long de $10 \mathrm{~m}$ présente une parfaite section ovoïde. À la Pinée, un puits de recherche s'enfonce verticalement sur $8 \mathrm{~m}$; preuve qu'il est possible de creuser vers le bas au moyen du feu.

\section{Le soutènement}

Dans les mines très anciennes, il est toujours délicat d'aborder ce sujet de manière frontale. En effet, les ouvrages les plus instables sont effondrés. Hors de l'eau, les bois de mine disparaissent en quelques siècles. Le remblaiement des chantiers restreint la vision. Le Fournel n'échappe pas à ces règles.

Deux exemples montrent que des étais en bois ont pu être installés pour soutenir le toit des chantiers, à moins qu'ils participaient à former des barrages pour contenir des remblais. On peut aussi observer des piliers en roche ou des restes de piliers qui ont été dépilés, soit par les Anciens, soit par les mineurs modernes. En règle générale, le soutènement actuel des chantiers instables est assuré par leur remblaiement.

\section{Les remblais}

Ils sont omniprésents et masquent la majeure partie des ouvrages souterrains. Leur nature reflète la prédominance de l'abattage par le feu caractérisé par un mélange de sables, de graviers, de blocs, et souvent par un litage fruste et lenticulaire, avec des charbons de bois dispersés ou concentrés (Marconnet, 2002, 2004; Py, 2009). Au Gorgeat, des infiltrations de boue rendent les coupes stratigraphiques difficiles à lire. À Saint-Roch, une grande coupe longitudinale de $10 \mathrm{~m}$ a pu être mise en relation avec l'avancement du front du chantier. Les déblais abandonnés dans la mine résultent d'un premier tri sur place. Certains lits de graviers peuvent néanmoins contenir une proportion notable de galène. L'importance de la partie stérile ou pauvre dans le volume de matériaux abattu et son foisonnement expliquent que le remblaiement des vides a été poussé à son maximum. Dans 
les travaux reculés de Saint-Roch, il ne reste qu'un étroit passage libre entre le faîte des chantiers et l'accumulation de remblais.

Dans les chantiers inclinés de Saint-Roch et de la Pinée, des murettes de blocs peuvent contenir ces masses de remblais et préserver des passages de circulation en fausses galeries (fig. 3) ou en coulisses. Mal construites, avec des petits blocs, ces murettes sont très mal conservées. Dans les chantiers verticaux de Combe Blanche, des planchers soutenant des remblais ont manifestement existé car d'importants soutirages indiquent des effondrements récents.

\section{L'aérage}

L'abattage par le feu engendre des fumées toxiques qu'il est impératif de maitriser et d'évacuer. Tous les réseaux souterrains profondément étendus présentent un système d'aérage. Il s'agit de galeries courant aux faîtes des chantiers verticaux ou au-dessus du plan des chantiers inclinés. Elles présentent un gabarit assez faible (inférieur à un mètre), sont parfois tortueuses, et sont enduites de suie. Elles se raccordent à un ouvrage montant, vertical ou très redressé, qui rejoint la surface. Les orifices de la plupart de ces puits d'aérage sont aujourd'hui comblés par des éboulis.

\section{La circulation et le transport}

Le chamboulement des remblais par les reprises d'exploitation et les éboulements ont en partie effacé les circuits de circulation au sein de l'exploitation médiévale. Il est cependant attesté que dans certains chantiers exigus, le déplacement des ouvriers était malaisé. Toutefois, beaucoup de galeries en roche et de fausses galeries réservées dans les remblais permettent une circulation debout, et les passages surbaissés ne semblent pas avoir été extrêmement fréquentés. Dans une galerie d'accès de Combe Blanche, des encoches attestent l'emplacement d'un plancher suspendu qui évitait aux mineurs de patauger dans les eaux d'écoulement.

Au Gorgeat, un ressaut vertical de deux mètres de hauteur était équipé de deux Kletterbaüme (échelles monoxyles) en place, et un autre Kletterbaum a été découvert dans des remblais (Ancel, 2008a). Ce type d'agrès pouvait équiper toutes les zones de circulation verticale. Les ouvrages inclinés comportent toujours des marches ou des gradins assez faciles à parcourir. Mais, on ne peut pas toujours leur donner un caractère purement intentionnel car leur aménagement est favorisé par la géologie.

On peut penser que l'évacuation du minerai et l'introduction du bois de chauffe se faisaient par portage au moyen

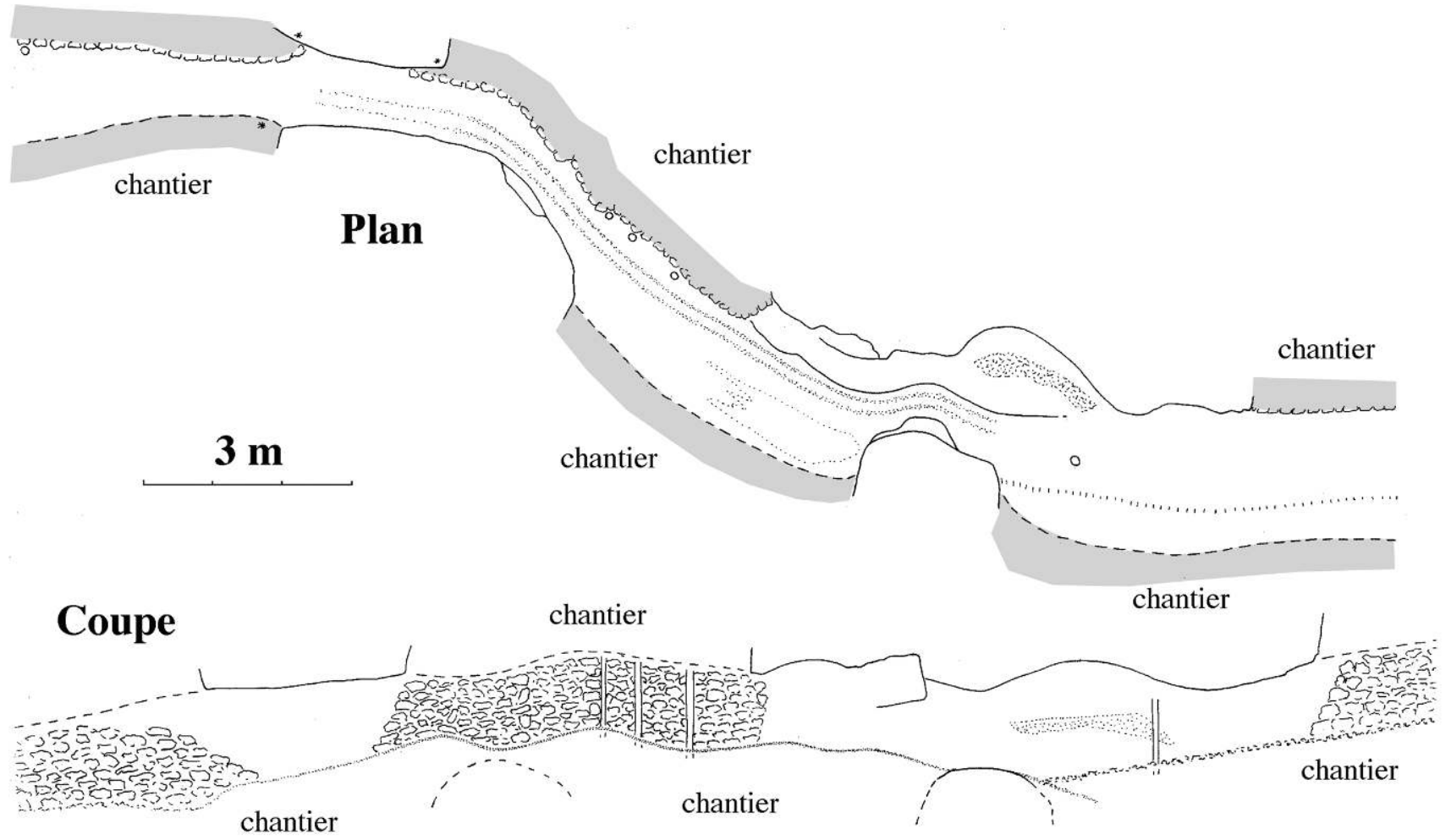

Figure 3 : Relevés en plan et en coupe de la voie de traînage de Saint-Roch.

Figure 3: Map and section of the sledging drift in St Roch sector. 
de sacs, éventuellement de hottes. Des fragments de toiles grossières ont été retrouvés dans les remblais. Pour les parties profondes, ce type de transport pouvait devenir fastidieux. Dans Saint-Roch, à $90 \mathrm{~m}$ du jour, on observe sur le sol d'une fausse galerie des traces de traînage (fig. 3). Il s'agit de deux sillons parallèles imprimés dans les remblais compactés, attestant le passage répété d'un engin large d'environ $30 \mathrm{~cm}$, muni de patins écartés de $17 \mathrm{~cm}$. Cette voie de traînage conservée sur $12 \mathrm{~m}$ de longueur, est sinueuse et ondulée, et présente localement des pentes pouvant atteindre $15^{\circ}$.

\section{L'exhaure}

L'escarpement des lieux, la fracturation du massif et sa décompression post-glaciaire ont favorisé le drainage naturel de la mine. Aucun ouvrage médiéval ne s'enfonce sous le niveau du torrent. L'exploitation du Gorgeat s'arrête au niveau du Fournel parce que le filon y disparait. Un rapport de la fin du XviII ${ }^{e}$ siècle signale à Saint-Roch des travaux presque entièrement inondés (Ancel, 2006b). L'examen actuel des lieux contredit cette assertion. Aujourd'hui tous les travaux anciens connus sont à sec. Ils sont drainés par des ouvrages anciens attestés ou supposés.

$\mathrm{Au}$ Gorgeat, une galerie de base se développe sur $45 \mathrm{~m}$ et draine les chantiers s'enfonçant depuis l'affleurement. Son creusement s'est effectué en plusieurs phases et des niveaux de drainage plus anciens montrant des pentes plus fortes - le niveau ultime présente une pente optimale de $0,5 \%$ - ont été mis en évidence (fig. 4). Ce complexe de proto-Erbstollen paraît avoir été improvisé à quelques mètres à peine sous la base d'une exploitation descendante.

Le niveau de base du réseau Saint-Roch et Combe Blanche n'a pas encore été retrouvé. Il existe pourtant car les chantiers profonds ne sont pas noyés. De plus, une galerie d'accès à ces chantiers présente une reprise de sa sole qui renvoie les eaux d'infiltration dans la mine, comme si on avait cherché à collecter un maximum d'eau avec cette galerie d'exhaure présumée. Son entrée serait située en contrebas de la crête de Saint-Roch sur un versant recouvert d'éboulis et de végétation. Une telle situation et sa longueur potentielle de plus de $50 \mathrm{~m}$, feraient de cet ouvrage un véritable Erbstollen.

\section{Les stratégies d'exploitation}

\section{La recherche des zones productives}

L'essentiel des panneaux minéralisés connus des Anciens affleurait en surface. Étant donnée la configuration des lieux, il fut donc aisé de les découvrir. Même lorsque la continuité des affleurements était interrompue par des placages d'éboulis dans les escarpements rocheux, il était très facile de purger et de nettoyer la roche.

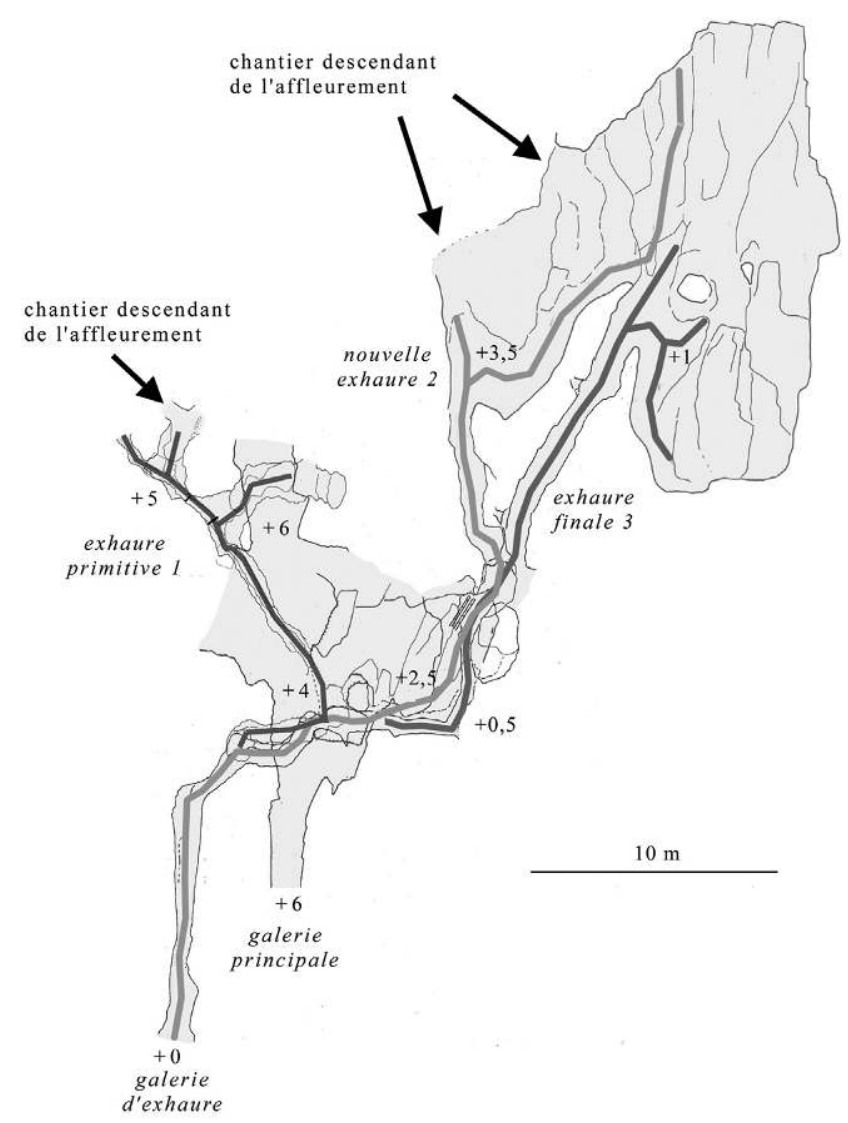

Figure 4 : (Voir planche couleur) Plan des galeries d'exhaure et des chantiers inférieurs du Gorgeat.

Figure 4: (See colour plate) Map of drainage adits and lower stopes in Gorgeat sector.

Certains panneaux décalés par un rejet de faille de quelques mètres ont été retrouvés par les Anciens. Des indices géologiques favorables - faille broyée facile à creuser, crochonnement des veines, entrainement du minerai dans le miroir de faille - ont peut-être encouragé les mineurs à tenter l'aventure. Cependant, au Gorgeat, les exploitants modernes ont pu retrouver un grand panneau vierge à moins d'un mètre des anciens travaux.

Le filon vertical de Combe Blanche n'affleure pas. Il se connecte à $80 \mathrm{~m}$ du jour avec la base du filon incliné de Saint-Roch. Il pourrait avoir été découvert par cette voie, car les datations radiocarbones indiquent une exploitation tardive (XIII ${ }^{\mathrm{e}}$ siècle). Mais l'analyse des ouvrages démontre une exploitation à partir de la zone proche du jour. Les mineurs ont suivi une faille de contact (quartzites/Verrucano) qui coupe le filon à $15 \mathrm{~m}$ de la surface. Cette faille a été sondée par trois niveaux de galerie indiquant que les Anciens avaient cherché quelque chose de précis dans ce secteur.

On connaît également des ouvrages s'enfonçant à l'écart des zones minéralisées connues : deux galeries engravées, 
supérieures à $5 \mathrm{~m}$; une descenderie de $15 \mathrm{~m}$; une descenderie/puits de $8 \mathrm{~m}$ et une "galerie/descenderie/puits » de $15 \mathrm{~m}$, c'est-à-dire un ouvrage qui commence horizontal, puis qui $s^{\prime}$ incline à $45^{\circ}$, et qui devient enfin vertical. Des circonstances particulières font que ces ouvrages sont restés visibles. Il peut en exister bien d'autres actuellement masqués par les éboulis. Un indice de puits a été fouillé en 2004 en aval de la Pinée. Il s'est avéré à peine profond de $4 \mathrm{~m}$ et a livré une galerie latérale de recherche longue de $6 \mathrm{~m}$.

\section{Le démarrage des exploitations}

Les panneaux minéralisés qui affleuraient en surface structurale ont été décapés comme dans une carrière à ciel ouvert, sans doute vers les $\mathrm{X}^{\mathrm{e}}$-XI ${ }^{\mathrm{e}}$ siècles. Ce fait rare a certainement contribué à dynamiser la mine de L'Argentière dès ses débuts, et ceci au vu et au su de tous. L'ébullition qui a régné sur les quartiers de la Pinée et de Saint-Roch était parfaitement perceptible depuis la plaine de la Durance, grande voie de circulation et de pèlerinage.

Les panneaux bien minéralisés à l'affleurement ont été exploités par des tranchées s'enfonçant de biais, ou verticalement. Mais, aujourd'hui, tous ces endroits sont comblés ou foudroyés. On peut observer des portions de tels chantiers proches du jour au sommet de Saint-Roch et à Lauzebrune où l'on constate une progression latérale et horizontale des fronts d'avancement; ce qui est somme toute logique pour des chantiers ouverts à partir d'un affleurement escarpé. Les tranchées qui auraient pu progresser en descente sont malheureusement inaccessibles actuellement. Ce cas de figure est cependant bien documenté sur le site-satellite de Faravel, où faute d'une extension du filon, l'exploitation n'a pas dépassé le stade de fosses peu profondes (Py et Ancel, 2007)

Le filon redressé du quartier Pinée Nord est par contre peu minéralisé à l'affleurement et l'on peut y observer différents ouvrages d'attaque - des descenderies et des puits - qui donnent rapidement accès à une zone d'exploitation remblayée. De courtes galeries, en allongement ou en travers-bancs, rejoignent la base de ces attaques, là où le filon est devenu exploitable. L'analyse des ouvrages démontre que ces galeries sont venues assister l'exploitation naissante qui a été initiée par les attaques sur l'affleurement (fig. 5).

\section{L'approfondissement des exploitations et le développement des ouvrages d'assistance}

Les exploitations du Gorgeat, de Saint-Roch, Combe Blanche et peut-être aussi celles de la Rouille et de la Pinée, se sont développées jusqu'à plus de $100 \mathrm{~m}$ du jour, sur une durée de deux à trois siècles ( $\mathrm{XI}^{\mathrm{e}}-\mathrm{XIII}{ }^{\mathrm{e}}$ siècles). Dans un tel contexte, l'espace souterrain utilisé pour la circulation, l'aérage et l'exhaure n'a pas dû se cantonner aux seuls chantiers d'exploitation, souvent exigus. Des ouvrages d'assistance ont été progressivement mis en place, généralement au plus près des chantiers d'abattage.

L'escarpement du relief favorise la circulation horizontale par des galeries (Ancel, 2000). Malgré les conditions sévères de remblaiements et de chamboulements tardifs, on observe les traces de niveaux de circulation sub-horizontaux qui traversent en galeries les zones stériles des filons, et en fausses galeries les zones remblayées des chantiers. Le transport des matériaux (minerais et bois de feu) y serait pratiqué au moyen de traîneaux. Il ne s'agit pas de niveaux tracés d'une traite du jour vers l'intérieur de la montagne, mais de portions de galeries et de fausses galeries aménagées en retrait $\mathrm{du}$ front de l'exploitation.

Les conduits d'aérage aménagés au faite des exploitations sont également en retrait des fronts d'exploitation. De courtes portions de galeries exiguës sont creusées les unes vers les autres à partir de points hauts des chantiers ou à partir de montages. Si nécessaire de nouveaux puits d'aérage sont creusés. On en compte trois successifs au faîte de Saint-Roch, toujours plus profonds évidemment. À Combe Blanche, un étroit puits de $12 \mathrm{~m}$, faisant la jonction entre une galerie de recherche et le chantier vertical, vient secourir l'exploitation (fig. 6). Au Gorgeat, un puits ouvert à $25 \mathrm{~m}$ des affleurements vient aérer le chantier incliné à $20^{\circ}$. De là, le réseau de galerie d'aérage s'étend sur $50 \mathrm{~m}$ communiquant en six points avec le front de l'exploitation. À mi-parcours, un montage aveugle semble préfigurer un orifice d'aérage qui aurait été avorté.

Près des affleurements, tous les chantiers sont drainés par de courtes galeries en zone stérile. Au Gorgeat, l'approfondissement du chantier entraîne un réajustement du circuit d'écoulement. Une galerie de $15 \mathrm{~m}$, ouverte au plus près du torrent, préfigure déjà ce que sera le niveau final. Mais, pour parer au plus pressé, un drain est aménagé dans la roche schisteuse, sous le chantier descendant, à partir de plusieurs petits puits distants à peine de 3 à $4 \mathrm{~m}$. Puis, la galerie est prolongée sur $30 \mathrm{~m}$ et atteint le point extrême du chantier, mais sa pente est exagérée. Alors, dans un troisième temps, la sole de la galerie est reprise. Ce surcreusement prend de l'ampleur et devient une nouvelle galerie qui au final permet de gagner 3,50 $\mathrm{m}$ de hauteur d'exhaure.

Ces ouvrages d'assistance organisent les exploitations souterraines (Ancel, 1998b) : niveaux de circulation au sein des chantiers; conduits d'aérage au-dessus des chantiers; galerie de drainage en dessous des chantiers. En zone stérile, des petits puits connectent ces ouvrages aux chantiers. On connait mal l'organisation verticale au sein des chantiers à cause de leur remblaiement, mais il semble qu'il existait des coulisses pour passer d'un niveau à l'autre. L'organisation 

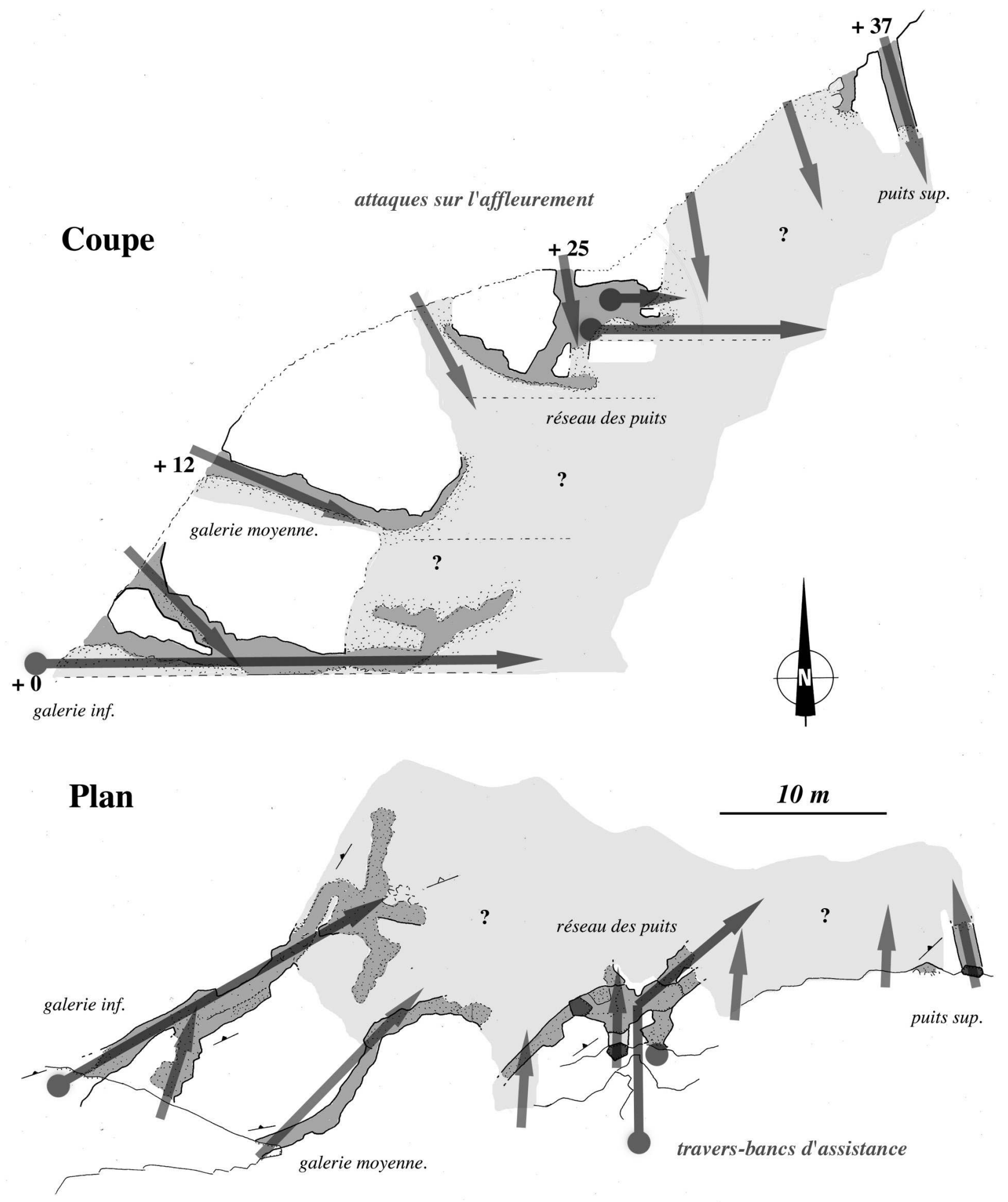

Figure 5 : Plan et coupe des attaques sur le filon de la Pinée. Figure 5: Map and section of early workings on the Pinée vein. 


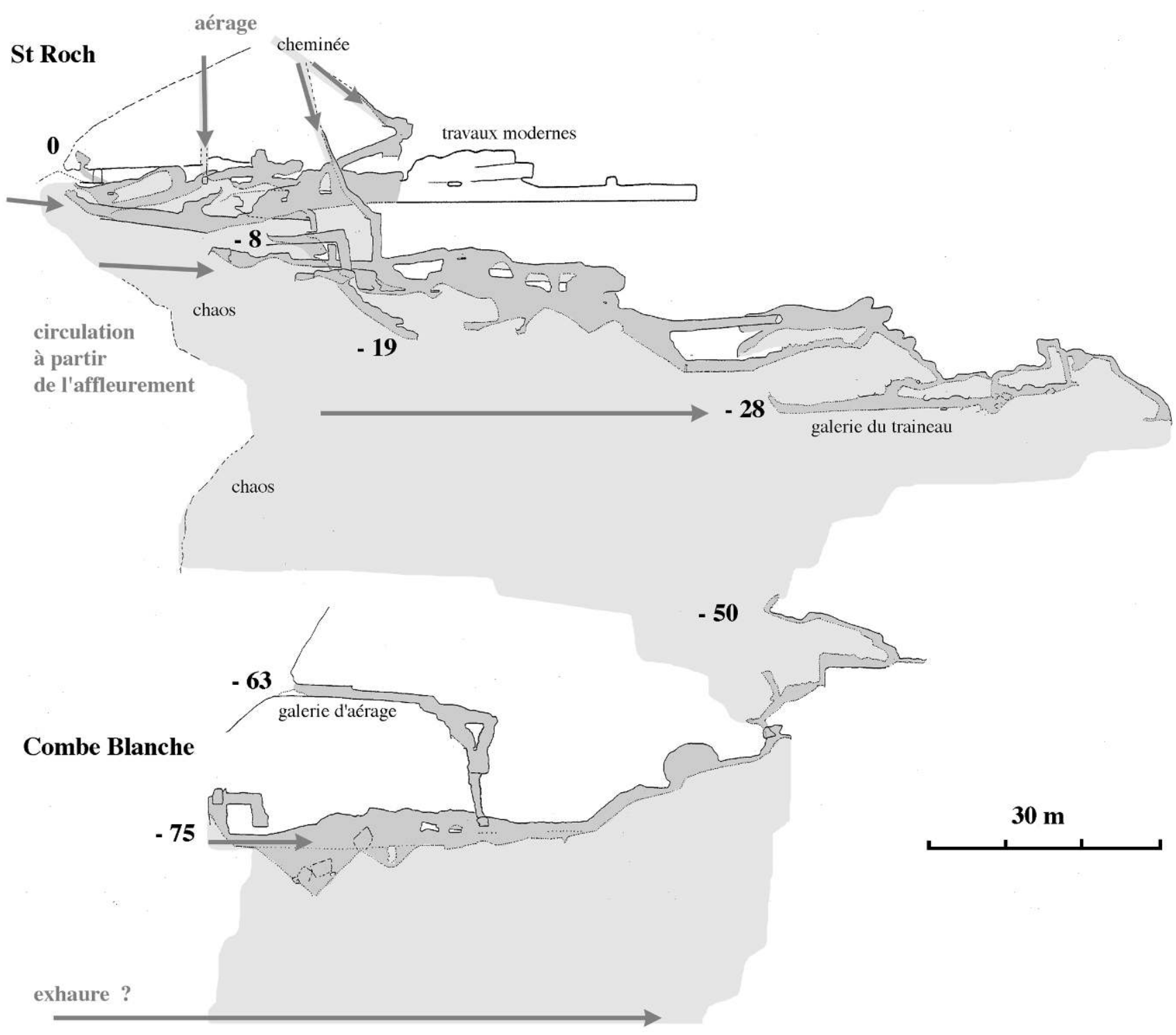

Figure 6: Coupe vertical des travaux de Saint-Roch et Combe Blanche.

Figure 6: Vertical section of the St Roch and Combe Blanche sectors.

de ces ouvrages préfigure d'une manière frustre le quadrillage de l'espace souterrain caractéristique des mines de la Renaissance (Ancel, 1990a). Les ouvrages d'assistance ne sont pas encore anticipés longtemps à l'avance, ils sont opportunistes et restent proches des chantiers. Ils sont aménagés au fur et à mesure des besoins, en utilisant au maximum des vides existants, en minimisant les trajets à percer, en restant par conséquent au plus près de l'exploitation.

Ainsi, au fur et à mesure que les fronts des chantiers s'enfoncent dans la montagne, l'exploitation s'organise tant bien que mal en retrait des fronts d'avancement, occasionnant probablement des suspensions d'activité pour cause d'en- fumage, d'engorgement ou d'ennoiement. Mais, tôt ou tard, les problèmes sont surmontés et l'exploitation ne sera véritablement stoppée que par l'épuisement des panneaux filoniens.

\section{LES INCIDENCES DE L'ABATTAGE PAR LE FEU}

La mine du Fournel renferme de nombreux témoignages de la technique d'abattage par le feu, aussi une recherche approfondie a été menée sur cette problématique (Ancel et Py, 2008; Ancel et al., 2009; Py, 2006; Py et Ancel, 2006, 2007). 


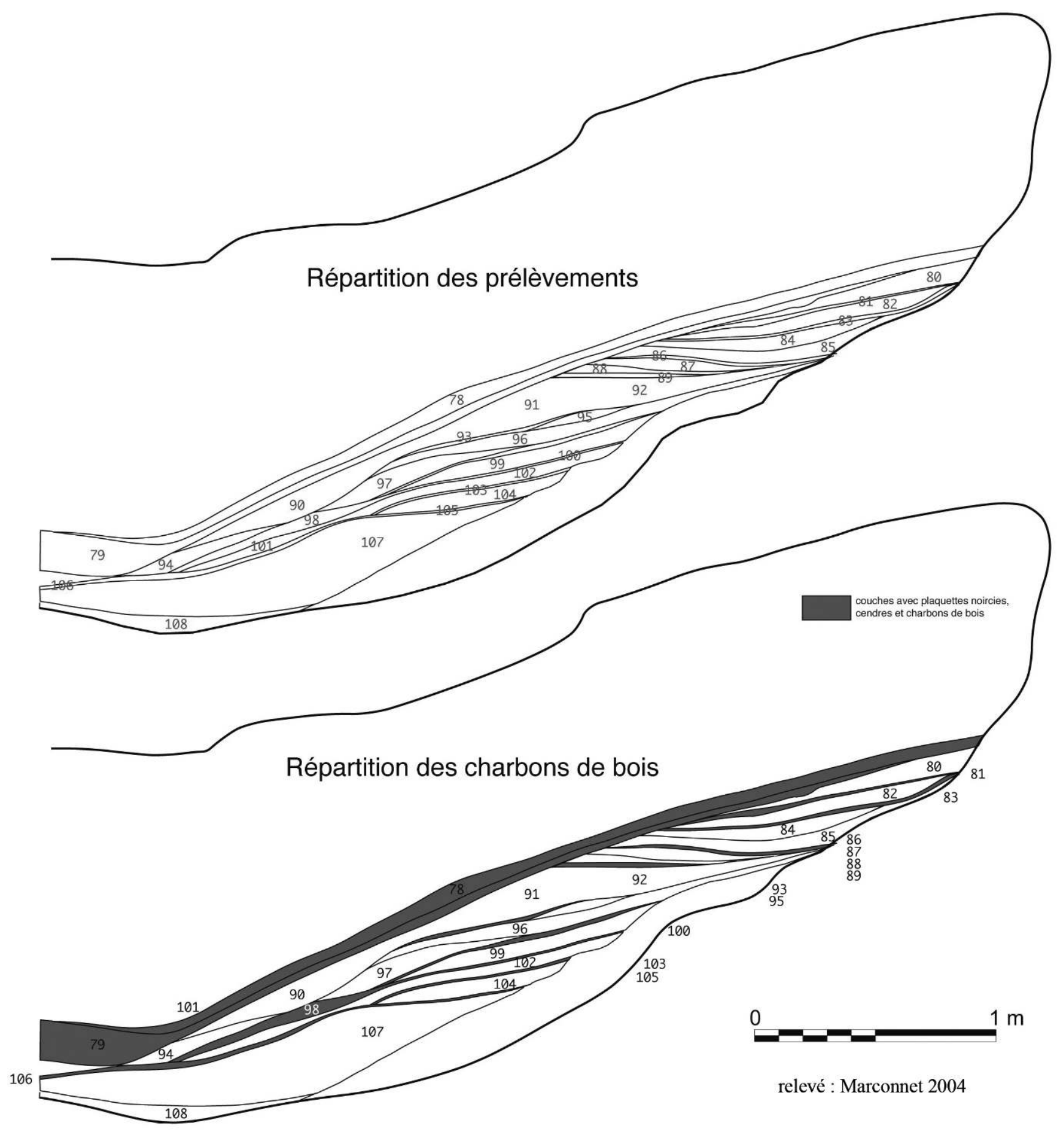

Figure 7 : Coupe stratigraphique dans des remblais du quartier Saint-Roch.

Figure 7: Stratigraphical cut through the backfill in the St Roch sector.

\section{Des textes à l'expérimentation}

Une enquête bibliographique a permis de recenser plusieurs centaines de références ayant trait à cette technique en contextes de mines, de carrières, de génie civil et de poliorcétique. Le corpus compte divers textes antiques, des règlements médiévaux, des ouvrages techniques modernes, des récits de voyageurs de toutes époques, etc., et la littérature 
scientifique contemporaine. Ces sources variées mettent bien en évidence les avantages et les inconvénients de l'usage du feu en mine - sévèrement réglementé au Moyen Âge - qui a perduré jusqu'au XIX ${ }^{e}$ siècle. Le recours à des aspersions d'eau fraîche est à présent clairement écarté de la pratique minière.

Les déblais accumulés sous terre et en surface sont les témoins précieux des milliers de bûchers embrasés durant plusieurs siècles. Un protocole d'étude de ces résidus a été mis en place pour caractériser la chaîne opératoire technique du feu, depuis le choix du combustible en forêt jusqu'au tri de la roche abattue sur le front de taille. Les coupes stratigraphiques dressées dans les remblais (fig. 7) permettent d'observer des successions de couches différentes définies par leur granulométrie, leur couleur et leur composition (minerai, encaissant, gangue, charbons de bois). Leur analyse minutieuse révèle la complexité du mode opératoire de l'abattage par le feu ponctué par des étapes de préparation du combustible, d'extraction au feu, à l'outil, de purge, de tri, de concassage et de déplacements des déblais judicieusement aménagés dans la mine de façon à faciliter la poursuite du travail.

Des expérimentations menées dans la mine du Fournel visent à retrouver la gestuelle et les modalités de cette technique, en caractérisant les paramètres qui entrent en jeu et en confrontant les données expérimentales aux observations archéologiques. Ces expériences sont réalisées dans une salle spacieuse, de façon sérielle, durant les hivers, période de l'année où le courant d'air est favorable à l'évacuation des fumées. L'objectif est de creuser une galerie dans les quartzites stériles, dépourvues de toute fracture, sans intervenir sur le feu après l'allumage. Après 200 feux, l'ouvrage mesure $1,60 \mathrm{~m}$ de hauteur et 2,20 $\mathrm{m}$ de profondeur. Plus de $3 \mathrm{~m}^{3}$ de roche ont été abattus $\left(9566 \mathrm{~kg}\right.$ ) et $45 \mathrm{~m}^{3}$ de bois secs ont été brûlés $(9556 \mathrm{~kg})$. Près de $200 \mathrm{~kg}$ de charbon de bois ont été produits, une proportion beaucoup plus importante que celle des remblais archéologiques, ce qui indique que ce sous-produit était récupéré pour d'autres usages.

L'évaluation des vides miniers nous permet d'avancer que le volume de minéralisation excavé au Moyen Âge est de l'ordre de $22000 \mathrm{~m}^{3}$. Le Fournel aurait pu produire environ 6000 tonnes de plomb et 15 tonnes d'argent. Il aurait été nécessaire de brûler de 100000 à 200000 stères de bois sec.

\section{La gestion forestière}

Les sources écrites étudiées (Py, 2009), principalement les textes législatifs de l'Occident médiéval, stipulent le droit d'exploiter tout le bois nécessaire pour l'extraction minière dans les forêts seigneuriales, privées ou communes, sous réserve du paiement d'une taxe, ou en toute liberté. Certains textes stipulent la mise à disposition des mineurs de réserves de boisements parfaitement délimitées. Les textes conservés concernant les territoires où s'étendent les prétentions delphinales suggèrent la mise à disposition pour la mine des «bois noirs " dont le dauphin se réserve la propriété, indépendamment de la propriété privée du terrain qui les recèle. Le terme nemora nigra est souvent interprété comme désignant les résineux, mais ces derniers ne forment pas tous des massifs " noirs ", c'est notamment le cas du Mélèze, résineux endémique des Alpes intra-alpines.

L'anthracologie révèle une exploitation presque exclusive des formations arborescentes caractéristiques des étages montagnard supérieur et subalpin inférieur avec la nette domination de Larix-Picea (Mélèze et/ou Épicéa) associé au Pin sylvestre et dans une moindre mesure au Sapin blanc avec un éventail peu ouvert de divers feuillus dont les fréquences absolues sont très faibles (Durand et Py, 2008; Py, 2009). Les analyses permettent néanmoins de percevoir en filigrane la végétation ligneuse des abords du site fortement anthropisés et dominés par les cultures jusqu'à plus de 1500-1600 m d'altitude. Ces boisements émiettés font l'objet de fagotages ponctuels pour le combustible d'allumage dont la production est dominée par le calibrage de bois de refend.

Les proportions des taxons identifiés et leurs variations, d'un secteur à l'autre et sur la longue durée, caractérisent l'état des boisements, l'évolution du territoire d'approvisionnement en bois de feu des mineurs et les modalités d'une gestion de la forêt pour la mine. Les mineurs ont massivement exploité le mélézin subalpin d'ubac associé au Sapin blanc dans sa limite inférieure dans les versants les plus frais et le mélézin de "descente » d'ubac. Le territoire d'approvisionnement parait ponctuellement orienté vers les massifs d'adrets d'altitude où les pinèdes de Pins sylvestres envahissent le mélézin diminué sous l'effet de son exploitation industrielle. Il se dégage du diagramme anthracologique de synthèse une gestion syncopée de ces boisements sur la longue durée probablement en relation avec l'évolution générale du couvert forestier et les besoins des autres activités artisanales et industrielles (fig. 8). La faculté du Mélèze à coloniser les espaces ouverts abandonnés à la déprise agraire de façon cyclique a certainement été mise à profit pour la mine. Les mineurs n'ont jamais manqué de bois et le faisaient venir des forêts dont la fourchette altitudinale peut se localiser entre 1600-1700 m et 1800-1900 m d'altitude. Les pourritures microscopiques carbonisées identifiées dans les fibres ligneuses attestent d'une phase de stockage pour le séchage du combustible calibré sur le carreau de la mine. 


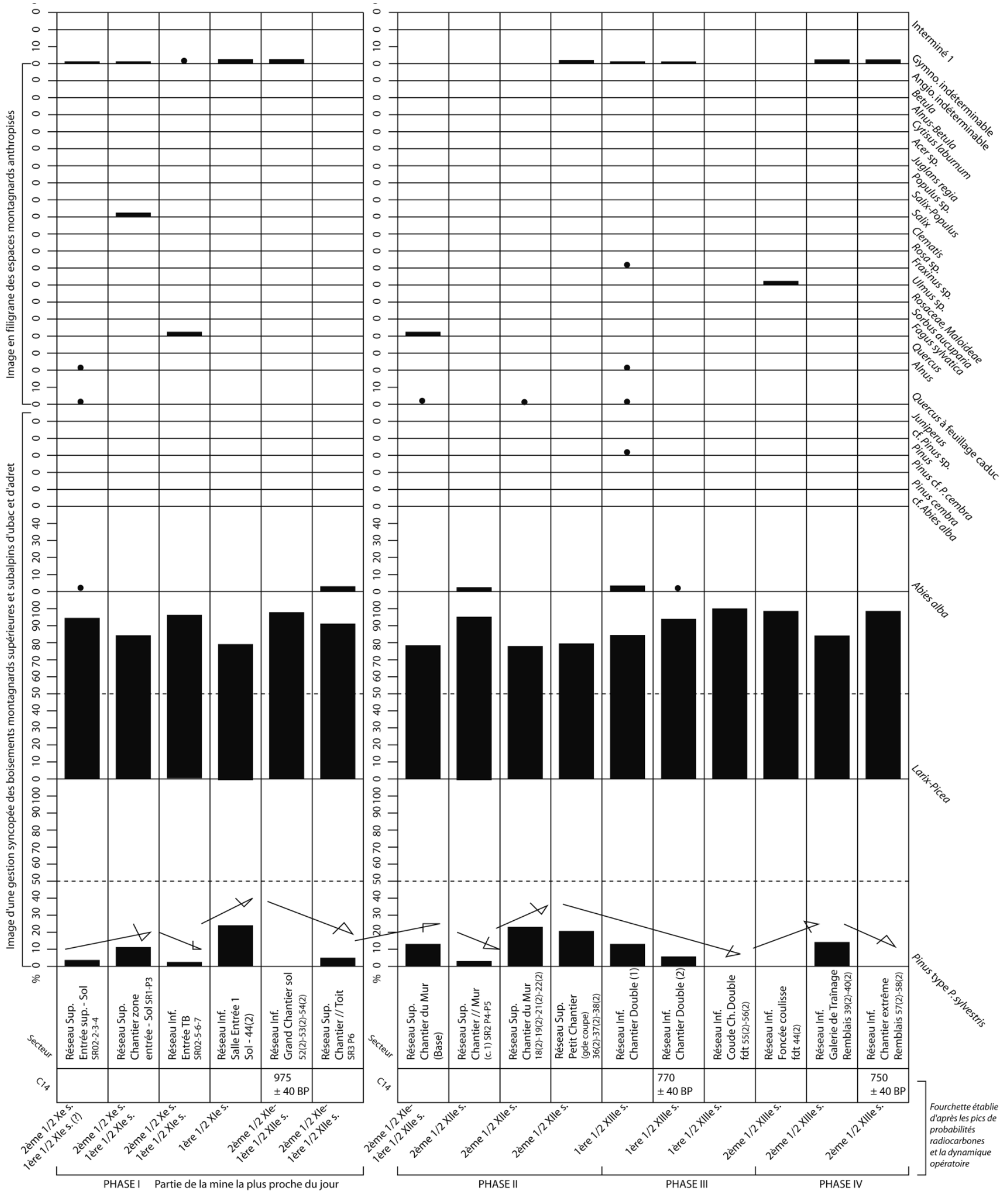

Figure 8 : Diagramme anthracologique de synthèse du quartier de Saint-Roch. Les lignes représentent les pourcentages de fragments par taxon. Les points représentent les pourcentages de fragments inférieurs à $0,5 \%$.

Figure 8: Anthracological diagram from the St Roch sector. The lines represent fragment percentage for each taxum. The points represent fragment percentage inferior to $0,5 \%$. 


\section{Discussion}

La mine médiévale du Fournel présente la plupart des caractéristiques des mines médiévales européennes, concernant les méthodes d'abattage, de tranport, d'aérage et d'exhaure (Ancel, 1998c; Bailly-Maître, 2002; Fluck, 2000; Rota et Tizzoni, 2006; Tizzoni, 1998). La bonne conservation des ouvrages et les fouilles extensives menées depuis 1992 permettent d'en avoir une vision assez représentative.

La puissance des filons étant peu élevée, les chantiers restent limités en épaisseur et ainsi les fonctions d'assistance ne peuvent y cohabiter. Ce n'est pas le cas des vastes exploitations de Vallauria (Tende) et de Largentière (Ardèche) où d'une immense salle longue de $160 \mathrm{~m}$, large de 20 à $30 \mathrm{~m}$, haute de $5 \mathrm{~m}$, émerge à peine un orifice d'aérage et un exutoire de drainage (Ancel, 1998b).

L'escarpement des lieux favorise un développement latéral de l'exploitation et une extraction horizontale des produits, au contraire des mines de Trento en Italie (Pospeny, 1880), de l'Altenberg à Sainte-Marie-aux-Mines (Fluck, 2000), de Melle en Poitou (Tereygeol, 2001) où l'extraction est essentiellement verticale, réalisée à partir d'une multitude de puits qui surplombent la couche exploitée (Pingenfeld) ou qui s'égrènent sur les affleurements (Pingenzug).

Le réseau minier montre une forme d'organisation dans l'espace et dans le temps (Ancel, 1998b) avec un développement certes limité des ouvrages d'assistance qui facilitent les fonctions de transport, d'aérage et d'exhaure, et qui souvent les éloignent de l'activité d'abattage du minerai. C'est une organisation discrète qui préfigure le système d'exploitation qui s'imposera avec la Renaissance dans les mines d'envergure, mais avec un certain retard pour les représentations iconographiques (Ancel, 1990b).

Les ouvrages d'assistance ne sont pas une innovation médiévale. Ils sont déjà présents et même parfois bien développés dans certaines mines de l'Âge du Fer et de l'Antiquité (Ancel et al., 1998; Domergue, 2008). Ils sont attestés pour le Haut Moyen-Âge, mais les exemples sont rares. L'exploitation verticale du Pontet à Bourg-d'Oisans (Ancel et Dardignac, 2008) est proche d'une falaise et les galeries d'aérage et d'exhaure sont réduites au minimum. Ces ouvrages semblent connaître un développement important seulement après le $\mathrm{XII}^{\mathrm{e}}$ siècle, mais encore faut-il considérer l'importance du contexte topographique et géologique. À Brandes (Bailly-Maître et Bruno Dupraz, 1994), la parfaite verticalité du filon fait confondre chantiers et ouvrages d'assistance, mais la fouille souterraine a mis en évidence un important niveau de drainage et de transport. À l'Altenberg (Fluck, 2000) des travers-bancs rejoignant les exploitations primitives par Pingenzug caractérisent une reprise tardive.
Deux exploitations des XIII ${ }^{\mathrm{e}}$-XIV ${ }^{\mathrm{e}}$ siècles présentent des organisations beaucoup plus complexes que celle du Fournel. La mine de Petra Alba à Saint-Laurent-le-Minier (Ancel, 2001; Bailly-Maître, 2002) exploite un lacis d'une cinquantaine de filons cuprifères. Les nombreux chantiers qui se recoupent en tous sens sont desservis par une quinzaine de niveaux de galeries et des puits intérieurs. Ce réseau s'organise dans des espaces concédés, conformément au règlement minier de 1228. Ici, la structure géologique complexe a encouragé le développement des galeries, dédiées d'abord à la recherche et l'exploration des minéralisations, puis à l'assistance des chantiers. À la mine de Castelminier d'Aulusles-Bains (Ancel, 2003), l'exhaure a été le problème majeur. Au cours de l'enfoncement de l'exploitation, plusieurs travers-bancs d'exhaure se sont succédés, toujours plus bas, toujours plus longs. Le dernier se développant sur $140 \mathrm{~m}$ aurait été inachevé à $20 \mathrm{~m}$ à peine des chantiers ennoyés.

Au Fournel, le développement limité des ouvrages d'assistance peut être justifié par le contexte topographique et géologique, mais en premier lieu par l'extrême dureté de la roche. Cette organisation opportuniste qui reste collée au gisement pourrait refléter un état d'esprit des exploitants qui n'encourageait pas les techniciens à tenter l'aventure à l'écart des filons et à se projeter dans le futur en anticipant les besoins potentiels de l'exploitation; un état d'esprit qui n'encourage pas les financiers à investir dans des ouvrages novateurs et coûteux, dont la nécessité n'est pas encore concrètement démontrée. Cette vision sera dépassée à la Renaissance.

\section{Bibliographie}

ANCEL, B. 1990a. L'organisation et dynamique des exploitations minières du Neuenberg au Xvi ${ }^{\mathrm{e}}$ siècle, in Benoît P. (dir.), Les techniques minières de l'Antiquité au XVIII siècle, CTHS, p. 421442.

ANCEL, B., 1990b. La représentation du milieu souterrain minier à travers l'iconographie du $\mathrm{Xv}^{\mathrm{e}}$ au XVII ${ }^{\mathrm{e}}$ siècle en province minière germanique, in L'image des mines et de la métallurgie du MoyenÂge à nos jours. Pierres et Terre, 33, p. 21-40.

ANCEL, B., 1998a. La mine du Fournel L'Argentière-la-Bessée, Hautes-Alpes, France : l'exploitation rationnelle aux $\mathrm{x}^{\mathrm{e}}$ $\mathrm{XIV}^{\mathrm{e}}$ siècles d'un filon de plomb argentifère, in Brigo L., Tizzoni M. (dir.), Il Monte Calisio. Atti del Convegno Europeo Civezzano-Fornace 1995, p. 161-193.

ANCEL, B., 1998b. Techniques minières et maîtrise de l'espace dans les mines d'argent médiévales. Exemples de mines de plomb argentifère des Alpes du Sud ( $\mathrm{x}^{\mathrm{e}}-\mathrm{XIV}^{\mathrm{e}}$ siècles), in Beck, P. (dir), L'innovation technique au Moyen Âge, éd. Errance, p. 108-110. 
ANCEL, B., 1998c. Les anciennes mines métalliques des Alpes du Sud, Archéologie et histoire en milieu souterrain, actes du Colloque Subterranéologie, Mons, Belgique, 1997, p. 51-63.

ANCEL, B., 2000. Les anciennes mines des Hautes-Alpes (Écrins, Queyras) et leur adaptation à l'environnement montagnard, in Boetsch, G. (dir.), Les écosystèmes alpins : approches anthropologiques, CRDP Aix-Marseille, p. 88-95.

AnCel, B., 2001. La mine de cuivre médiévale de Petra-Alba (Saint-Laurent-le-Minier) : Relevés du réseau souterrain et interprétation de la dynamique opératoire, L’Argentière-laBessée, Rapport CCSTI.

AnCel, B., 2003. Exploration et relevés archéologiques des ouvrages miniers anciens du Castelminier à Aulus-les-Bains, in Tereygeol, F. et al. (dir.), Le Castel-Minier (Aulus-les-Bains), Rapport d'activité archéologique, CNRS UMR 5060, p. 40-82.

ANCEL, B., 2006a. La mine d'argent du Fournel à L'Argentière-laBessée Hautes-Alpes : méthodologie et bilan 1991-2001, in Barge, H. (dir.), Mine et métallurgie en Provence et dans les Alpes du Sud de la Préhistoire au XX siècle : reconversion industrielle et enjeux culturels, Châteaudouble 2001. -4000 ans d'histoire des mines. Mélanges Jean-Paul Jacob, Actilia Multimédia, p. 71-85.

ANCEL, B., 2006b. La mine d'argent du Fournel à travers les sources historiques : 1 . Le souvenir de l'exploitation médiévale, Cahiers du Château Saint-Jean, 1, p. 3-24.

AnCEL, B., 2008a. Les vestiges en bois de la mine du Fournel à L'Argentière-la-Bessée Hautes-Alpes, in BAILlY-Maître, M.-C. et al. (dir.), Archéologie et paysage des mines anciennes : de la fouille au Musée, Picard, p. 75-87.

ANCEL, B., 2008b. Le paysage minier en zone de montagne : l'exemple des Hautes-Alpes, in Bailly-Maître M.-C. et al. (dir.), Archéologie et paysage des mines anciennes : de la fouille au Musée, Picard, p. 233-247.

Ancel, B. et Dardignac, C., 2008. La mine de plomb du Pontet à Bourg d'Oisans, Cahiers du Château Saint-Jean, 5, p. 97-157.

Ancel, B. et Cowburn, I., 1998. L'Argentière-la-Bessée : un CCSTI au service du patrimoine minier, L'Archéologie Industrielle, CILAC, 31, p. 44-47.

Ancel, B., Marconnet, C., Kinchin-Smith, R. et Granger, L., 1996. La mine d'argent du Fournel, La publication archéologique sur CD Rom. Exemples pratiques d'écritures éléctroniques, Ministère de la Culture, dossier $\mathrm{n}^{\circ} 12$.

Ancel, B., Morin, D., Tizzoni, M., Kammenthaler, E. et Cottet, M., 1998. La mine de cuivre de Campolungo, une exploitation révolutionnaire de l'Âge du Fer, Notizie Archeologiche Bergomensi, 6, p. 163-184.

AnCel, B. et Py, V., 2008. L'abattage par le feu : une technique minière ancestrale, Archéopage, 22, p. 34-41.

Ancel, B., Py, V. et Marconnet C., 2009. De l'usage minier du feu : à l'interface homme et environnement. Sources et expérimentations, in Durand, A., Jockey, Ph. (dir.), Mélanges offerts à Georges Comet, Cahiers d'Histoires des Techniques 8, Presses Universitaires de Provence.

Bailly-Maître, M.-C., 2002. L'argent : du minerai au pouvoir dans la France médiévale, coll. "Espace Médiévaux », Picard.

Bailly-Maître, M.-C. et Bruno Dupraz, J., 1994. Brandes-enOisans, La mine d'argent des Dauphins (XIt-XIV siècle), Isère, Documents d'Archéologie en Rhône-Alpes, p. 9.

BRGM, 1997. Étude géologique et structurale de l'ancienne mine de plomb argentifère du Fournel à L'Argentière-la-Bessée (Hautes-Alpes), Rap. BRGM R 39625.

BRGM, 2004. Étude de la mine de L'Argentière (Hautes-Alpes), Rapport annuel des travaux effectués en appui aux études d'archéologie minière dans le cadre de la convention nationale SDABRGM. Rap. BRGM/RP-53585-FR, p. 13-30

Cowburn, I., 2008. La gestion des vestiges des sites miniers : l'exemple des mines d'argent du Fournel, in Bailly-Maître, M.-C. et al. (dir.), Archéologie et paysage des mines anciennes : de la fouille au Musée, Picard, p. 157-163.

Domergue, C., 2008. Les mines antiques : la production des métaux aux époques grecque et romaine, Picard.

Durand, A., 2007. Savoir-brûler, savoir gérer le combustible chez les potiers et les mineurs médiévaux méridionaux, Bilans de l'ACI, Histoire des Savoirs, p. 37-48.

Durand, A. et Py, V., 2008. L'évolution des écosystèmes dans le haut Champsaur et la montagne de Dormillouse (HautesAlpes, France) au crible des charbons de bois archéologiques, in Bernardi, Ph. (dir.) Forêts alpines et charpentes en Méditerranée, Éditions du Fournel, p. 32-35.

FuUcK, P., 2000. Sainte-Marie-aux-Mines : les mines du rêve, éd. Patrimoine Minier.

Grandemange, J., Coluin, Y. et Martaud, A., 1990. Problème de la superposition de trois phases d'exploitation dans un faisceau filonien : l'exemple de la mine Sapin-Vert, in Benoît, P. (dir.), Les techniques minières de l'Antiquité au XVII' siècle, CTHS, p. 421-442.

Marconnet, C., 2002. Étude sédimentologique des résidus de l'abattage au feu issus des expérimentations et des mines médiévales $d u$ Fournel, Rapport de Consultant, Saint-Pierre-le-Chastel.

Marconnet, C., 2004. Etude sédimentologique des résidus de l'abattage au feu issus des mines médiévales du Fournel, Rapport de Consultant. Saint-Pierre-le-Chastel.

Posepny, F., 1880. Über den alten Bergbau von Trient, Archiv für praktische Geologie, 1, p. 1-121.

Py, V., 2004. Protoindustries et agropastoralisme : approche diachronique de l'anthropisation des montagnes : prospection du Vallon du Fournel et de ses alentours, Bulletin Scientifique du SRA PACA 2003, p. 52-54.

Py, V., 2006. Mine charcoal deposits : methods and strategies. The medieval Fournel silver mines in the Hautes-Alpes (France), in A. Dufraisse (éd.), Firewood economy: analytical tools and methods, British Archaeological Reports, 2006, p. 35-46. 
Py, V., 2009. Mine, bois et forêt dans les Alpes du Sud au Moyen Âge. Thèse de doctorat, Université d'Aix-Marseille I.

Py, V. et AnCel, B., 2006. Archaeological experiments in firesetting : protocol, fuel and anthracological approach), in Dufraisse A. (éd.), Firewood economy : analytical tools and methods, British Archaeological Reports, p. 43-54.

Py, V. et Ancel, B., 2007. Exploitation des mines métalliques de la vallée Freissinières (Hautes-Alpes, France) : contribution à l'étude de l'économie sud-alpine aux $\mathrm{IX}^{\mathrm{e}}$-XIII ${ }^{\mathrm{e}}$ siècles, Preistoria Alpina, 42, p. 83-93.
Rota, C. et Tizzoni, M., 2006. Le miniere, in Tizzoni M. et al., Alle origini della siderurgia lecchese, Materiali I. Musei Civici di Lecco, p. 33-39.

Tereygeol, F., 2001. Les mines d'argent carolingienne de Melle, Thèse de doctorat, Université de Paris I.

Tızzoni, M., 1998. Le miniere di argento medievali in Lombardia in Brigo L., Tizzoni M., Il Monte Calisio, atti del Convegno Europeo Civezzano-Fornace (1995), p. 269-279. 